\title{
Is the class A macrophage scavenger receptor (SR-A) multifunctional? - The mouse's tale
}

\author{
Nick Platt and Siamon Gordon \\ Sir William Dunn School of Pathology, University of Oxford, Oxford, United Kingdom \\ Address correspondence to: Nick Platt, Sir William Dunn School of Pathology, University of Oxford, South Parks Road, Oxford OX1 3RE, \\ United Kingdom. Phone: 44-1865-275533; Fax: 44-1865-275515; E-mail: Nick.Platt@pathology.ox.ac.uk.
}

J. Clin. Invest. 108:649-654 (2001). DOI:10.1172/JCI200113903.

The class A macrophage scavenger receptor (SR-A) is the prototypic member of an expanding family of membrane receptors collectively termed scavenger receptors (SRs) (1-3). Receptors of this group recognize a number of ligands, including chemically modified or altered molecules and, in particular, the modified lipoproteins that are pertinent to the development of vascular disease. As shown in Table 1, all characterized SR-A ligands are polyanionic, although many polyanions fail to bind SR-A. Here, we wish to summarize and comment upon the evidence for SR-A being both a multiligand and multifunctional receptor, considering both the available in vitro data and the results of several studies of SR-A-deficient mice. Two other SRs, namely SR-B1 (Krieger, ref. 4) and LRP (Herz and Strickland, ref. 5), are the subject of separate reviews in this Perspective series.

Two themes that can be drawn from studies of SRs as a whole are particularly relevant to this discussion. First, because these receptors display broad and seemingly overlapping ligand-binding properties, biological specificity is likely to be determined not only by ligand structure and the signal transduced following ligand binding, but also by other considerations. These include the distribution and availability of the various SRs, their ability to interact with other receptors, and their relative affinities for the various ligands. Second, the apparent redundancy of ligand binding is achieved despite the absence of conserved protein sequences among the distinct classes of SRs. A mechanistic understanding of individual SRs' broad yet specific ligand recognition and of the features shared by these unrelated molecules will require the resolution of tertiary structures of multiple SRs.

The structural basis of ligand binding by SR-A As shown in Figure 1, SR-A is a multidomain trimeric molecule composed of three identical protein chains. The Class A SRs comprise SR-A types I and II, the nonfunctional splice variant type III, and a distant receptor called MARCO (2). These molecules each contain a characteristic collagenous domain, and binding studies on a series of truncation and point mutant receptors show that the collagenous region of SR-A is required for ligand recognition, at least for binding modified lipoproteins such as oxidized or acetylated LDL (6). This domain has therefore been the focus of mutagenesis approaches to attempt to define the structural basis of broad ligand binding.

The original structure-activity investigation demonstrated that a cluster of four lysines in the most C-terminal portion of the receptor is the ligand-binding site, and that within this motif, lysine-337 of bovine SR-A is essential for lipoprotein recognition. These results were interpreted in a computer model prediction of SR-A structure in which these residues formed a positively charged groove responsible for the binding of the negative charges on ligands. A subsequent, more extensive mutagenesis study has shown the contribution of other residues spanning the entire collagen domain, which were revealed when binding assays were performed at $4^{\circ} \mathrm{C}(7)$. These studies suggested high affinity recognition is dependent upon conformational interactions between distinct domains and the contact of residues on them. This is consistent with a model deduced from the demonstration of a possible hairpin configuration for the receptor, in which the collagen domain is folded back against the $\alpha$-helical coiled-coil domain, rather than being fully extended (8). Given this structural model, lig-

\section{Table 1}

SR-A ligands

\section{Ligand}

Oxidized Low Density Lipoprotein (LDL) Acetylated LDL

Oxidized High Density Lipoprotein (HDL) Maleylated bovine serum albumin (BSA) Malondialdehyde BSA

Fucoidan

Dextran Sulfate

Polyguanylic acid (poly G)

Polyinosinic acid (poly I)

Crocidolite asbestos

Silica

Lipopolysaccharide (LPS)

Lipoteichoic acid (LTA)

Gram negative bacteria

Gram positive bacteria

Apoptotic cells 


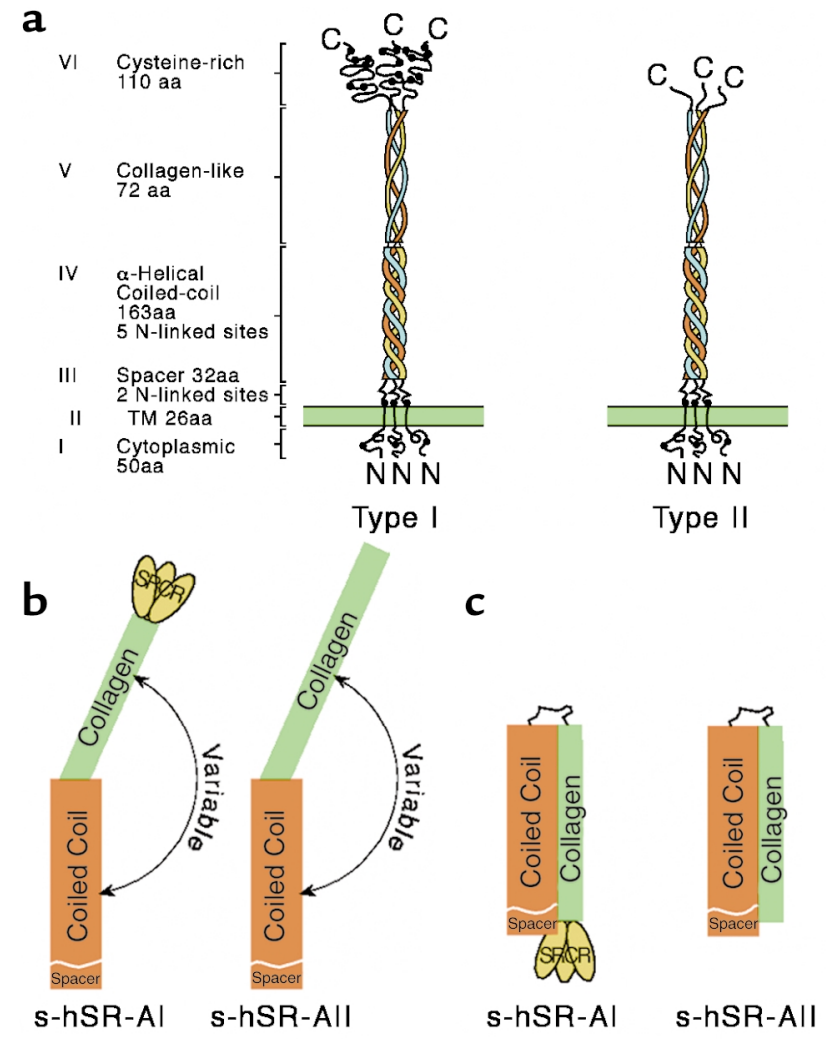

Figure 1

Structures of the class A macrophage SR. (a) Domain organization of type I and type II receptors. Extended (b) and compact (c) forms of soluble receptor have been seen by rotary shadowing and negative staining (8). Adapted, with modifications from refs. 8 and 37 (3).

and affinities may depend on the regulated folding of the receptor, which would influence the extent of contact between domains. The construction of suitable mutants that either promote or prevent flexible articulation of the receptor should permit testing of this hypothesis. It would be of interest to examine whether similar binding properties are observed with ligands other than AcLDL, or if configuration has a greater or lesser effect on their recognition. Interestingly, a naturally occurring mutation of SR-A in the C57/BL6 mouse strain has recently been described, which has been identified as amino-acid changes in the $\alpha$-helical coiled-coil domain that affect receptor immunoreactivity, but apparently not function $(9,10)$. Detailed kinetic studies of this or other sequence variants may reveal important clues to the relationship between ligand binding and the receptor's conformational state.

SR-A shares with SR-B1 (see Krieger, this Perspective series, ref. 4) an unusual binding property referred to as nonreciprocal cross-competition of ligands (1). This term is used to describe a situation in which ligand $\mathrm{A}$ can completely compete for the binding of ligand $\mathrm{B}$, but the latter cannot effectively displace the former. For example, although oxLDL inhibits the binding of acLDL by SR-A totally, acLDL can only block that of oxLDL partially. The structural basis of this pattern of ligand binding is unclear, although it has been interpreted as reflecting the existence of two discrete but overlapping ligand binding sites on the receptor. Nonreciprocal interactions have been observed so far only in experiments with cultured cells, but the prospect that they occur in vivo raises exciting possibilities. In particular, such a situation would favor the hierarchical and sequential interactions of the receptor with different ligands and thus might account for some of the biological complexity of SR function. For instance, under some circumstances, SR-A might colocalize with a known ligand but fail to bind it because another ligand is bound preferentially, even though the latter interaction may be of similar or lower affinity. In order to define the receptor-ligand events fully, it will be necessary to identify all the types of SRs and characterize all the ligands that are present in any given biological context.

With the cloning of the gene for the bovine SR-A, two transcripts were identified encoding different forms of the receptor (1). Identical isoforms have been shown in the subsequent cloning of the murine, human, and rabbit homologues (11). Type I SR-A is distinguished by the presence of an additional C-terminal region, the SR cysteine-rich (SRCR) domain. Not only has this domain been conserved in the SR-As of other mammals, it has been found in a number of proteins across different phyla (12). Because it has not so far been shown to influence the binding of modified lipoprotein, the presence of SRCR-like motifs is not predictive of SR activity. However, its conservation implies an unidentified function. The potential identification of molecules that bind specifically to the SRCR domain and not the collagenous region could reveal significant biological interactions, but to date, with a few relatively minor exceptions, the binding properties of type I and type II SR-A are currently considered identical. The recent generation of a mouse lacking type I but retaining type II expression may clarify the role of the SRCR (M. de Winther and M. Hofker, personal communication).

Although other SRs have been identified in invertebrates, no obvious homologues of SR-A are found in the fully sequenced genomes of Caenorbabditis elegans and Drosophila melanogaster. Therefore, the evolutionary origin of the SR-A isoforms is uncertain. The gene appears to be common among the vertebrates, as related expressed sequence tags have been reported for zebrafish and Xenopus, but SR-A homologues in more primitive chordate species have not yet been reported.

\section{Analyses of SR-A-deficient mice}

Although its activities are well-defined in vitro, the contributions of SR-A in vivo have been difficult to determine. The existence of other receptors that display very similar activities and the lack of reagents to effectively distinguish between them, make defining specifics very challenging. Most of the known ligands do not distinguish between receptors, so a detailed comparison of ligand profiles would be needed to infer the identity of a particular SR. Fortunately, specific antibodies can now be used to define the cellular expression, distribution, and abundance of the known SRs, and even to suggest previously unrecognized activities for these molecules. The $\mathrm{mAb}$ $2 \mathrm{~F} 8$, which is directed against the murine SR-A, revealed a role for this receptor in macrophage $(\mathrm{M} \phi)$ adhesion. We 
have used this $\mathrm{mAb}$ to follow SR-A expression, and we found it widespread among discrete $M \phi$ populations in vivo, including those in the spleen, thymus, liver, heart, and gut $(13,14)$. It is restricted to mature $M \phi$ and is not expressed by monocytes (N. Platt and S. Gordon, unpublished observations). These observations might therefore suggest either a function common to almost all $\mathrm{M} \phi$ or multiple contributions unique to particular cells.

Unfortunately, because it apparently clears rapidly from the bloodstream, mAb 2F8 did not prove useful as an inhibitor of function in vivo (S. Gordon, et al., unpublished observations). Rather, as is now almost universal for investigations of biological function, the production of mice genetically deficient in SR-A has paved the way for whole-animal experimentation. Phenotypic analysis of the $S R-A^{-/-}$mouse currently allows the most sophisticated investigations into the various roles of SR-A and it is timely to evaluate these studies.

\section{Atherogenesis in SR-A-deficient mice}

Despite broadening of the activities with which SR-A is now associated (Figure 2), studies of its involvement in vascular pathologies remain dominant because of the relationship to disease. To begin to evaluate the roles of SR-A, Suzuki and colleagues generated a mouse deficient in both isoforms of the receptor (15). Isolated peritoneal $\mathrm{M} \phi$ from these animals showed a significant decrease in the endocytic degradation of acLDL (> 80\%) and oxLDL (> 30\%). This was in accordance with a series of previous in vitro data that collectively demonstrated SR-A-mediated uptake of modified lipoproteins by $M \phi$, leading to the formation of lipid-laden foam cells (1). To study the contribution of SR-A to atherosclerosis, Suzuki et al. crossed these mice with atherosclerosis-prone apoE-null animals. The resulting doubly deficient mice had moderately increased serum cholesterol and significantly reduced lesion formation (on the order of 60\%) compared with apoE-null controls, confirming that SR-A promotes atherosclerosis (15). However, the work in other models has raised doubt about the generality of this finding. On the LDL receptor-deficient background, which promotes susceptibility to diet-induced disease, the absence of SR-A only results in a marginal lesion reduction (16); for mice on the apoE3Leiden transgenic background, which are hypercholesterolemic and susceptible to diet-related disease, atherosclerotic pathology is actually enhanced in the absence of SR-A (17). In a reciprocal experiment, crossing LDL receptor-null mice with a transgenic strain that overexpresses SR-A lead to reduced atherosclerosis (17).

Is it possible to reconcile these apparent discrepancies? If, for the sake of argument, we discount the possibility that the pathologies differ mechanistically in the different experimental systems, the simplest explanation is that SR-A plays multiple roles in atherogenesis. Some activities, such as the endocytosis of modified lipoproteins, may promote disease, whereas other, currently undefined activities may be antiatherogenic. Subtle differences in disease progression between the various models may therefore involve SR-A at different sites or in different biological contexts. SR-A may also function differentially depending on an animal's genetic background, as has been suggested from a recent study discussed by Mazzone (18). Finally, a caveat that applies to knockout studies in general is worth considering here. Since knockout mice lack the targeted gene product not only in the tissue studied, but globally and throughout development, they have ample opportunities to alter the expression of other functionally related genes. Thus, these studies may reveal more about molecular plasticity or the ability of mice to compensate developmentally for the absence of a specific protein than about the role of the protein in normal adult tissues. If such compensatory changes vary among the different mutant and transgenic lines, the different phenotypes seen may not strictly reflect the lack of SR-A, but also the consequences of all the resulting changes in gene expression. Until these issues are resolved, the potential utility of drugs that perturb SR-A activity to prevent vascular disease remains problematic.

Although Kupffer cells and the sinusoidal endothelium express SR-A, and isolated cells prepared from $S R-A^{-/-}$mice show reduced endocytic activity, the clearance of exogenous lipoprotein is identical in livers of wild-type and receptor-null animals (19). Because greater than $90 \%$ of the material is removed within 5 minutes of introduction, the capacity of the organ may be too great for detection of the SR-A-dependent component. This apparent excess of general SR activity is a theme that
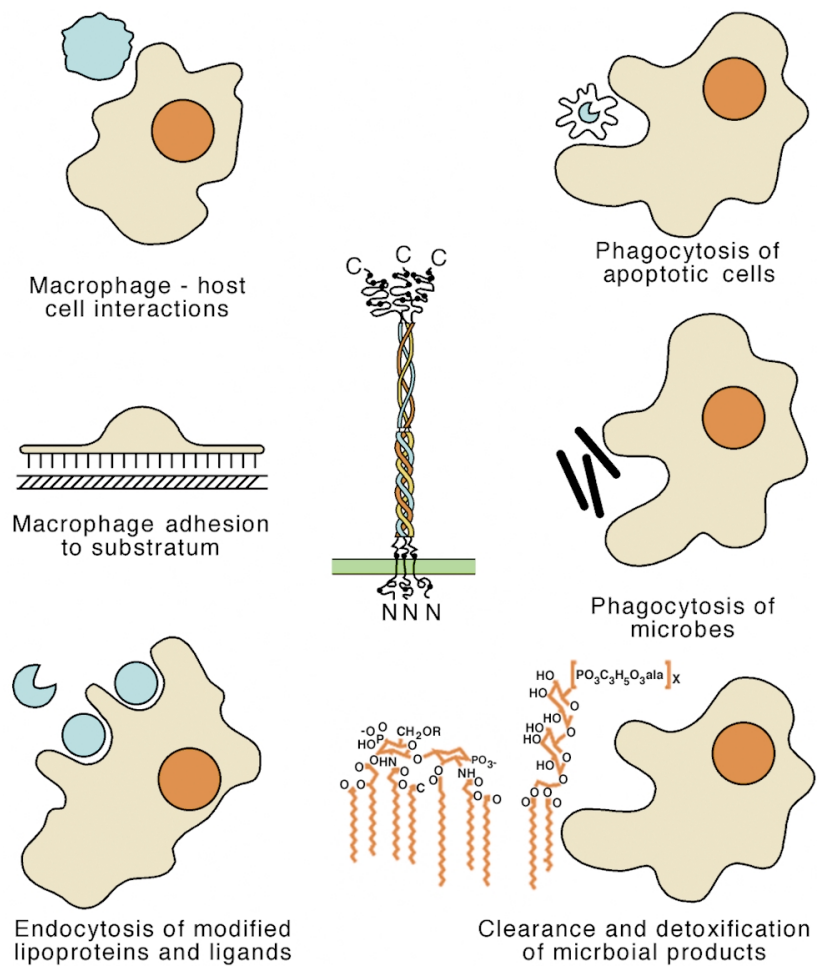

Figure 2

Putative biological roles for SR-A. In vitro studies that have examined the breadth of SR-A activities, together with analyses of the SR-A-deficient mouse, have suggested a number of different biological roles for the receptor. These include the endocytosis of modified lipoproteins and its association with vascular disease; the adhesion of $M \phi$ to the substratum and other populations of cells; the binding and ingestion of microbes; the detoxification of pathogen-specific components; and the phagocytosis of apoptotic or unwanted host cells. 
occurs repeatedly, perhaps because current experimental designs are inadequate. Alternatively, it may be that SR activities are so important, it is essential to have a surplus. The identification of new SRs and their manipulation in vivo should help resolve these possibilities.

\section{M $\phi$ adhesion}

Recruitment and retention at relevant tissue sites is a prerequisite for the appropriate $M \phi$ function. This is true for both resident tissue populations and activated cells that migrate towards sites of infection and inflammation. SR-A-mediated M $\phi$ adhesion was first demonstrated with the isolation of the $2 \mathrm{~F} 8 \mathrm{mAb}$, which inhibits a cation-independent, serum-dependent component of adhesion (13). SR- $A^{-/-}$peritoneal cells also display reduced adhesion and spreading over the first 24 hours after isolation (15), although they subsequently adhere normally. We have detected no obvious absence of any specific tissue $\mathrm{M} \phi$ populations or altered retention of activated cells in either untreated animals or bacillus Calmette-Guerin (BCG) granulomatas of SR-A-null mice. van Velzen and colleagues have reported a reduction in Kupffer cell number compared with wild-type (20). However, there is no evidence yet of affected adhesion in vivo or resultant altered liver physiology. Mato's cells, which are granular epithelial cells of the glial limitans of neural tissues, fail to extend and develop properly in $S R-A^{-/-}$ mice, perhaps because of impaired adhesion (21).

SR-A-dependent adhesion may only be of significance in certain pathological states, when normal molecules are suitably modified to become ligands for SR-A, thus promoting unwanted cell retention. In particular, glycated ECM components, which accumulate with age and in conditions including diabetes, serve as adhesive ligands for SR-A in vitro (22). A further link is suggested by the observation of accelerated atherosclerosis in diabetic individuals, but to date studies of an appropriate model formally demonstrating an interaction in vivo have not been reported.

\section{Host defense and innate immunity}

The tenet that SR-A is an element of the innate immune system developed principally from two lines of evidence. First, binding studies of SR-A demonstrated that molecules derived from certain pathogens, specifically, LPS of Gram negative and lipoteichoic acid of Gram positive bacteria compete for the binding of other known ligands $(1,2)$, suggesting that SR-A could function as a pattern recognition receptor, activating innate responses to conserved bacterial structures. Second, SR-A is expressed primarily by $\mathrm{M} \phi$, which are among the first line of antimicrobial defense (14). Suzuki et al. originally reported that $S R-A^{-/-}$mice have impaired protection against infection by Listeria monocytogenes and herpes simplex virus (15); subsequently, Thomas et al. (23) confirmed increased sensitivity to Staphylococcus aureus because of impaired clearance of bacteria. Recently, a defect in the uptake of Neisseria meningitidis in vitro has been shown in $S R-A^{-/-}$bone marrow culture-derived M $\phi$ (S. Gordon et al., unpublished observations). However, the nature of the protective mechanisms provided by SR-A against these very different pathogens is not obvious and could vary with the microbe. More detailed analyses of the underlying process in infected $S R-A^{-/-}$mice should be informative with regard to the diversity of pathogens and surface components that are able to bind to SR-A. A receptor that could both recognize a number of different infectious agents and engage disparate anti-microbial effector systems would be a valuable component of protection against the evolving pressures of pathogens.

Uncontrolled cellular activation, a dangerous consequence of disseminated infection and the release of microbial components, can lead to toxic shock and septicemia. Haworth et al. (24) tested the in vivo relevance of the data of Hampton and coworkers, which showed that SR-A can mediate clearance and detoxification of plasma endotoxin (25). Following LPS challenge, BCGprimed SR-A deficient mice are more susceptible than similarly treated wild-type mice to endotoxic mortality caused by overproduction of TNF- $\alpha$ and IL- 6 . Consistent with Hampton's in vitro work, these data suggest that SR-A-dependent uptake of LPS does not activate, and might even suppress, cytokine responses to this bacterial component. However, recently, the opposite findings have been reported, namely that $S R-A^{-/-}$mice are more resistant to endotoxic shock (26). Although there are several possible explanations for these contradictory results, one attractive idea, drawn from the different experimental protocols used in the two series of experiments, is that the effect of LPS binding to SR-A is determined by the extent of activation of the M $\phi$. Such sensitivity to cellular physiology might add a further dimension to the roles of this multifunctional receptor.

\section{Adaptive responses}

As with M $\phi$ and other cells of the myelomonocytic lineage, SR-A may function in both the innate and the adaptive branches of the immune response. Evidence is now emerging for a role for SR-A in antigen presentation. It has been recognized for some time that the acquired immune system can effectively respond to modified antigens capable of interacting with SRs (2). Alteration of molecules into structures that can bind SRs can induce responses that may be enhanced in comparison with those elicited by the corresponding native antigen. However, the precise identity of the SRs involved has remained obscure. Nicoletti et al. (27) have recently reported significantly diminished responses to maleylated albumin in $S R-A^{-/-}$mice, but at this time we can only speculate as to the precise mechanism and the cells responsible. However, these findings are potentially useful therapeutically, as they may indicate a route for promoting antigen delivery within vaccination strategies.

\section{Disorders of the nervous system}

SR-A is expressed on microglia in the CNS under certain conditions (28), raising the possibility that it may be actively involved in the development of some neuronal disease. Perhaps the most interesting studies have shown a relationship with the etiology of neurodegeneration in Alzheimer disease (AD). Prominent expression of SR-A has been shown on reactive microglia surrounding amyloid plaques (29). Cell-culture studies have demonstrated that the endocytosis of the key $\mathrm{A} \beta$ peptide is mediat- 
ed by SRs (30), and that microglial adhesion to amyloid fibrils (31) and the consequent production of neurotoxins are attributable to SR-A. Therefore, SR-A may enhance pathogenesis by helping to establish or to maintain the characteristic plaques and the deleterious production of reactive species. On the other hand, the potential for SR-A-dependent endocytic clearance of $A \beta$ from the brain could slow plaque formation and thus be protective. Lack of the receptor does not lead to any obvious defect in brain development or to signs of pathology in $S R-A^{-/-}$animals. Therefore, in order to examine the consequences of the absence of SR-A, Huang et al. (32) bred receptor-deficient mice with a transgenic line that expresses human amyloid precursors (hAPP), in which amyloid is deposited and synaptic degeneration observed. In their experiments, hAPP-transgenic $S R-A^{-/-}$ animals showed no clear reduction or promotion in plaque accumulation and concomitant degeneration, suggesting that SR-A does not play a unique pathological role in amyloidosis (32). These observations are more in line with the induction of SR-A by adverse conditions, as was true in a previous study in which only LPS-activated microglia expressed the receptor. Because the hAPP-transgenic mouse model shows AD-associated pathological changes but no other disease symptoms, it may not provide an ideal model in which to evaluate all relevant effects of the absence of SR-A.

\section{Phagocytosis of apoptotic cells}

The burgeoning interest in the process of apoptosis has relatively recently extended to the fate of cells committed to die. In vivo, apoptotic or senescent cells are rapidly phagocytosed, frequently by $M \phi$, via mechanisms that do not provoke inflammation (33). As discussed by Fadok et al. (this Perspective series, ref. 34), several SRs accounted for the different phagocytic activities. In fact, apoptotic cell recognition may be a universal property of SRs, because it has been demonstrated for all those receptors that have been examined so far, including invertebrate ones (3). It is tempting therefore, to propose that this is their ancestral function, because of the early evolutionary emergence of the process.

SR-A has been implicated by in vitro experimentation in the recognition of at least two populations of dying cells - apoptotic thymocytes and activated platelets (33). In both cases the anti-SR-A mAb 2F8 significantly inhibits ingestion by activated $M \phi$. Furthermore, the impaired recognition of apoptotic thymocytes seen in experiments with $S R-A^{-/-}$thymic $\mathrm{M} \phi$ is comparable to that achieved through $\mathrm{mAb}$ blockade of wild-type cells. However, detailed scrutiny of thymocyte clearance under normal and enhanced apoptotic cell load failed to reveal an obvious phagocytic deficit in vivo (34). This observation, like the other reports that do not shown phenotypic alterations in the absence of SR-A, could be due to imprecise methodology that is unable to detect a small or transient difference, or to the ability of other receptors to compensate fully. SR-A may well not be uniquely important for this process in the thymus. More recent work, however, indicates that phagocytosis of apoptotic neutrophils in situ occurs inefficiently in SR-A-deficient mice (N. Platt et al., unpublished observations).

\section{Is SR-A multifunctional?}

Given the many disparities between in vitro and in vivo findings and even between different studies in $S R-A^{-/}$ mice, it remains likely but is still not proven that this receptor is multifunctional. Others have described knockout mice generated by homologous recombination as both panacea and frustration, which might apply to $S R-A^{-/-}$animals. Although in most cases, studies of knockout mice have produced results consistent with what might have been predicted from in vitro activities, sometimes overtly similar, but subtly different approaches have yielded conflicting data. Because separate research groups have not performed identical investigations it is not always easy to determine a consensus view. In the case of $S R-A^{-/}$mice, such apparent discrepancies could be interpreted as reflecting the biological complexity and multiple functions of this receptor. The demonstration of a protective role for SR-A in host defense, with which almost all of the reports are in agreement, offers up a separate dilemma: How does SR-A prevent infection by such an array of pathogens? Again, it could be explained by either a single, unifying mechanism or several separate activities.

\section{Why multiple ligands for SR-A?}

The most intriguing and defining property of SR-A is its unique pattern of ligand-binding activities. Together with its expression on the ubiquitous and highly versatile $M \phi$, the diversity of ligands would seem to predict a multitude of functions for this molecule (35). Conversely, the reason $\mathrm{M} \phi$ can perform multiple tasks is in part because of their expression of molecules such as SR-A. We might wonder whether this strategy of employing a single receptor to fulfil several requirements is advantageous compared with the situation of having many receptors, each performing more restricted functions. It is not obvious that there is a disadvantage to the latter, because the vast majority of plasma membrane receptors are very narrow in the range of molecules with which they can interact. Typically they have a limited number of ligands. There does not seem to be any sort of theoretical limit on the generation of receptor diversity. Even when inherited material becomes limiting, genetic recombination has facilitated greater variation in specific cases (e.g., immunoglobulins). Interestingly, other receptors that are part of the innate system, such as complement receptors, $\mathrm{CD} 14$, toll-like receptors, and the $\mathrm{M} \phi$ mannose receptor may also interact with a relatively wide range of (nonoverlapping) ligands $(2,36)$. Therefore, evolutionary pressure may have favored the emergence of membrane molecules with this property, before subsequent surges in receptor diversity, after which they remained sufficiently important to ensure their conservation.

SR-A differs fundamentally from most receptors with respect to the chemical and physical forms of its ligands. Recognition by ligand by the latter usually depends upon a defined structure that is encoded directly by the amino-acid sequence of the protein. Even with respect to nonpeptide recognition, such as that of carbohydrate structures, there is a probable limit to diversity, because specific transferases and glycosidases generate the sugar structures. In contrast, ligands of SR-A are for the most part covalently altered molecules that are distinguished 
from the native molecule in part by charge. These modifications, such as oxidation and glycation, may arise from either enzymatic or nonenzymatic reactions $(1,2)$. Recognition is restricted to specific modifications. While the amino-acid sequence of the native molecule may ultimately limit what can be modified, the extent and forms of modification are potentially quite variable. For instance, minimally and moderately oxidized species of LDL probably interact with separate receptors and induce different biological outcomes.

Since oxidation and the other events occur frequently in tissues, SR ligands are likely to be widespread, although they may be particularly prevalent under conditions such as cellular stress, damage, aging, and death. The chemical modifications that render a native molecule recognizable by SRs may therefore be seen as markers of unwanted potentially dangerous molecules and cells and direct them for removal and disposal. This may be the common link. Thus, Krieger and colleagues have very entertainingly described SR-A as behaving as "molecular flypaper" (1). Although some of the ligands that have been identified are relevant to specific pathologies (or, in the case of microbial products, associated with disease-causing agents) the majority are nonphysiological, and there is a dearth of identified endogenous ligands. It is possible that a major function of SR-A has not been analyzed because of our lack of knowledge of the identity of naturally occurring ligands within hosts.

The challenge remains to explain the occurrence of other receptors whose activities apparently overlap with SR-A to such a large extent. This may be due to volume of biological need and the requirement for excess capacity to avoid potentially harmful situations. We currently have much greater knowledge of the shared properties of SRs than of those that are specific to distinct receptors. Continued study of $S R-A^{-/-}$mice will help us understand not only what this particular receptor does, but also what it does not do.

\section{Acknowledgments}

The authors would like to thank Rosangela da Silva and Hugh Perry for comments and ideas on the manuscript. We apologize to those authors whose work could not be cited as the original publication, owing to space constraints. The authors would like to thank the British Heart Foundation and the Medical Research Council for financial support.

1. Krieger, M., and Herz, J. 1994. Structures and functions of multiligand lipoprotein receptors: macrophage scavenger receptors and LDL receptorrelated protein (LRP). Annu. Rev. Biochem. 63:601-637.

2. Pearson, A.M. 1996. Scavenger receptors in innate immunity. Curr. Opin. Immunol. 8:20-28.

3. Platt, N., and Gordon, S. 1998. Scavenger receptors: diverse activities and promiscuous binding of polyanionic ligands. Chem. Biol. 5:R193-R203.

4. Krieger, M. 2001. Scavenger receptor class B type I (SR-BI) is a multiligand high density lipoprotein receptor that influences diverse physiologic systems. J. Clin. Invest. In press.

5. Herz, J., and Strickland, D.K. 2001. LRP: a multifunctional scavenger and signaling receptor. J. Clin. Invest. In press.

6. Doi, T., et al. 1993. Charged collagen structure mediates the recognition of negatively charged macromolecules by macrophage scavenger receptors. J. Biol. Chem. 268:2126-2133.

7. Andersson, L., and Freeman, M.W. 1998. Functional changes in scavenger receptor binding conformation are induced by charge mutants spanning the entire collagen domain. J. Biol. Chem. 273:19592-19601.

8. Resnick, D., Chatterton, J.E., Schwartz, K., Slayter, H., and Krieger, M. 1996.
Structures of class A macrophage scavenger receptors. Electron microscopic study of flexible, multidomain, fibrous proteins and determination of the disulfide bond pattern of the scavenger receptor cysteine-rich domain.J. Biol. Chem. 271:26924-26930.

9. Fortin, A., Penman, M., Stevenson, M.M., Krieger, M., and Gros, P. 2000. Identification and characterisation of naturally occurring variants of the macrophage scavenger receptor (SR-A). Mamm. Genome. 11:779-785.

10. Daugherty, A., Whitman, S.C., Block, A.E., and Rateri, D.L. 2000. Polymorphism of class A scavenger receptors in C57BL/6 mice. J. Lipid Res. 41:1568-1577.

11. Freeman, M., et al. 1990. An ancient, highly conserved family of cysteine-rich protein domains revealed by cloning type I and type II murine macrophage scavenger receptors. Proc. Natl. Acad. Sci. USA. 87:8810-8814.

12. Resnick, D., Pearson, A., and Krieger, M. 1994. The SRCR superfamily: a family reminiscent of the Ig superfamily. Trends Biochem. Sci. 19:5-8.

13. Fraser, I., Hughes, D., and Gordon, S. 1993. Divalent cation-independent macrophage adhesion inhibited by monoclonal antibody to murine scavenger receptor. Nature. 364:343-346.

14. Hughes, D.A., Fraser, I.P., and Gordon, S. 1995. Murine macrophage scavenger receptor: in vivo expression and function as receptor for macrophage adhesion in lymphoid and non-lymphoid organs. Eur. J. Immunol. 25:466-473.

15. Suzuki, H., et al. 1997. A role for macrophage scavenger receptors in atherosclerosis and susceptibility to infection. Nature. 386:292-296.

16. Sakaguchi, H., et al. 1998. Role of macrophage scavenger receptors in dietinduced atherosclerosis in mice. Lab. Invest. 78:423-434.

17. de Winther, M.P., van Dijk, K.W., Havekes, L.M., and Hofker, M.H. 2000. Macrophage scavenger receptor class A: a multifunctional receptor in atherosclerosis. Arterioscler. Thromb. Vasc. Biol. 20:290-297.

18. Mazzone, T. 2000. Scavenger receptors in atherosclerosis. New answers, new questions. Arterioscler. Thromb. Vasc. Biol. 20:2506-2508.

19. Ling, W., et al. 1997. Oxidized or acetylated low density lipoproteins are rapidly cleared by the liver in mice with disruption of the scavenger receptor class A type I/II gene. J. Clin. Invest. 100:244-252.

20. van Velzen, A.G., Suzuki, H., Kodama, T., and van Berkel, T.J. 1999. The role of scavenger receptor class $A$ in the adhesion of cells is dependent on cell type and cellular activation state. Exp. Cell Res. 250:264-271.

21. Mato, M., Ookawara, S., and Sakamoto, A. 1997. Growth retardation of Mato's fluorescent granular perithelial (FGP) cells in scavenger receptor knockout (SRKO) mice. Anat. Rec. 247:307-316.

22. El Khoury, J., et al. 1994. Macrophages adhere to glucose-modified basement membrane collagen IV via their scavenger receptors. J. Biol. Chem. 269:10197-10200.

23. Thomas, C.A., et al. 2000. Protection from lethal gram-positive infection by macrophage scavenger receptor-dependent phagocytosis. J. Exp. Med. 191:147-156.

24. Haworth, R., et al. 1997. The macrophage scavenger receptor type A is expressed by activated macrophages and protects the host against lethal endotoxic shock. J. Exp. Med. 186:1431-1439.

25. Hampton, R.Y., Golenbock, D.T., Penman, M., Krieger, M., and Raetz, C.R. 1991. Recognition and plasma clearance of endotoxin by scavenger receptors. Nature. 352:342-344.

26. Kobayashi, Y., et al. 2000. Role of macrophage scavenger receptor in endotoxin shock. J. Pathol. 192:263-272.

27. Nicoletti, A., et al. 1999. The macrophage scavenger receptor type A directs modified proteins to antigen presentation. Eur. J. Immunol. 29:512-521.

28. Bell, M.D., et al. 1994. Upregulation of the macrophage scavenger receptor in response to different forms of injury in the CNS. J. Neurocytol. 23:605-613.

29. Christie, R.H., Freeman, M., and Hyman, B.T. 1996. Expression of the macrophage scavenger receptor, a multifunctional lipoprotein receptor, in microglia associated with senile plaques in Alzheimer's disease. Am. J. Pathol. 148:399-403.

30. Paresce, D.M., Ghosh, R.N., and Maxfield, F.R. 1996. Microglial cells internalize aggregates of the Alzheimer's disease amyloid beta-protein via a scavenger receptor. Neuron. 17:553-565.

31. El Khoury, J., et al. 1996. Scavenger receptor-mediated adhesion of microglia to beta-amyloid fibrils. Nature. 382:716-719.

32. Huang, F., et al. 1999. Elimination of the class A scavenger receptor does not affect amyloid plaque formation or neurodegeneration in transgenic mice expressing human amyloid protein precursors. Am.J. Pathol. 155:1741-1747.

33. Platt, N., da Silva, R.P., and Gordon, S. 1998. Recognizing death: the phagocytosis of apoptotic cells. Trends Cell Biol. 8:365-372.

34. Fadok, V.A., Bratton, D.L., and Henson, P.M. 2001. Phagocyte receptors for apoptotic cells: recognition, uptake, and consequences. J. Clin. Invest. In press.

35. Platt, N., Suzuki, H., Kodama, T., and Gordon, S. 2000. Apoptotic thymocyte clearance in scavenger receptor class A-deficient mice is apparently normal. J. Immunol. 164:4861-4867.

36. Aderem, A., and Ulevitch, R.J. 2000. Toll-like receptors in the induction of the innate immune response. Nature. 406:782-787.

37. Rohrer, L., Freeman, M., Kodama, T., Penman, M., and Krieger, M. 1990. Coiled-coil fibrous domains mediate ligand binding by macrophage scavenger receptor type II. Nature. 343:570-572. 